\title{
IoT FOR NEXT GENERATION
}

\author{
Dr. G.P.Hegde \\ Department of ISE \\ SDMIT, Ujire, Karnataka, India
}

Abstract - Information technology and information science is a fast growing area due to rapid growth of next generation expectations leads to internet of things (IoT) which can connect us various devices and things at any instant. Controlling of different electrical and electronic appliances or machines through internet is made possible by IoT. This paper mainly focuses on history, structure, present and next generation status of IoT, and technology challenges of IoT. This paper also deals about applications in various fields where IoT roles up its different issues. The state of art approaches illustrates how IoT meets the various challenges about past and future technology.

Keywords - Internet, device, things, generation, sensor

\section{INTRODUCTION}

The Internet of Things (loT) is the network of substantial objects or "things" embedded with electronics, computer software, sensors, and network connectivity, which enables these objects to collect and exchange data. IoT is an advanced technology which the industries are starting to adapt into their operation. IoT is simply a user interface that a user can integrate into almost all the electrical/ electronic devices to transform them into a smarter one.

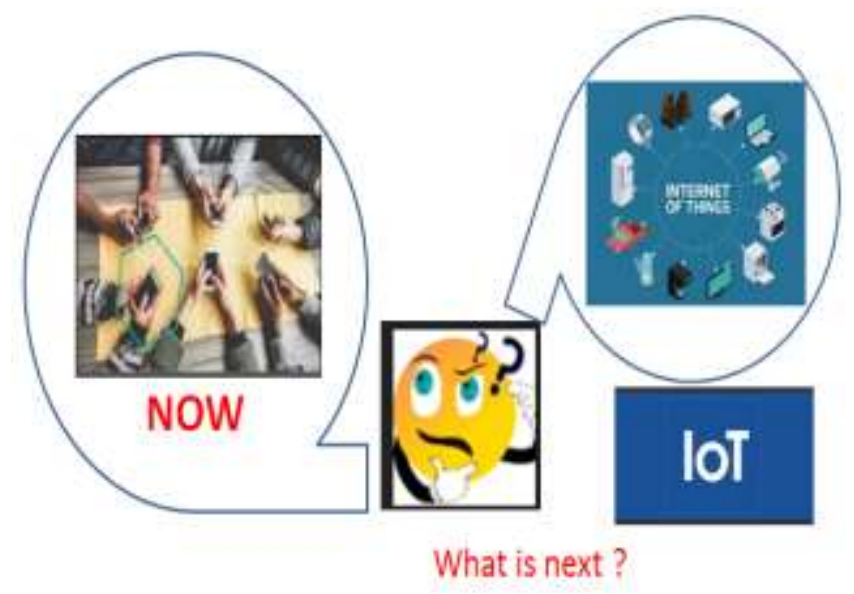

Fig. 1. Representation of think for next generation

Figure 1 shows people are thinking prototype of internet has been grown up to internet of things. So IoT is an integral part of next generation internet.

\author{
Deekshith K \\ Department of ISE \\ SDMIT, Ujire, Karnataka, India
}

Studying the IoT as a whole requires knowledge from many technical disciplines, including distributed systems, mobile computing, human-computer interaction, cloud computing, artificial intelligence and data semantics, as well as many nontechnical disciplines, and the many business, domestic and personal fields to which the IoT is or will be applied.

This next generation technology is also preferred by many industries for the variety of applications it offers for a user like real-time analytics, examination of collected data, cloud data storage, activate an stroke based on a value an user sets, remote announcement etc. A "Thing" in the context of the (loT), is an entity or substantial object that has a unique identifier, an embedded system and the ability to transfer data over a network. These devices collect useful data with the help of various existing technologies and then autonomously flow the data between other devices. The authors in [1] developed an electronic information desk system. In this system author used SMS based approach in different way. In [2] (20020the purpose of research is to identify with the feasibility of IoT in bus transportation system in Singapore. The Singapore, which is technically advanced but still, has scope of improvement in their transportation system. Using the IoT for the consumer to understand and evaluate different bus options in an efficient manner.

The rest of the paper is organized as follows. Framework of IoT is illustrated in section II. Hardware of IoT are presented in section III. Applications of IoT are mentioned in section IV. Concluding remarks are given in section $\mathrm{V}$.

\section{FRAME WORK OF IOT}

\section{A. History of IoT-}

"Internet of Things" was stated by Kevin Ashton in 1999. Ashton who was working in supply chain optimization, wanted to attract senior management's attention to a new exciting technology called RFID. Because the internet was the hottest new trend in 1999 and because it somehow made sense, he called his presentation "Internet of Things". IoTenabled applications have become part of larger business objectives, enabling digital transformation with a focus on new services and applications. He quoted IoT as next generation of internet. 
International Journal of Engineering Applied Sciences and Technology, 2019

Vol. 4, Issue 3, ISSN No. 2455-2143, Pages 153-156

Published Online July 2019 in IJEAST (http://www.ijeast.com)

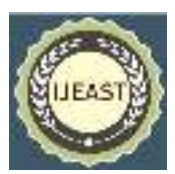

\section{$B$. How IOT works}

The Internet of Things (loT), also sometimes referred to as the Internet of Everything (loE), consists of all the web-enabled devices that collect, send and act on data they attain from their surrounding areas and environments using embedded sensors, processors and communication hardware. These devices, often called "connected" or "smart" devices, can sometimes talk to other related devices, a process called machine-to-machine (M2M) communication, and act on the information they get from one another. Humans can interact with the gadgets to set them up, give them instructions or access the data, but the devices do most of the work on their own without human intervention. Their existence has been made possible by all the tiny mobile components that are available these days, as well as the always-online nature of our home and business networks. Figure 2 shows basics steps followed during construction of IoT system.

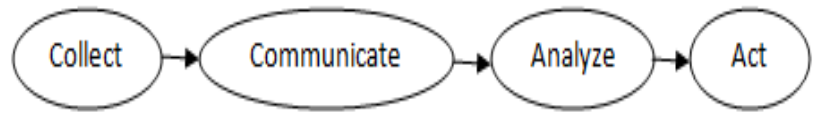

Fig. 2. Steps involved in working of IoT

Various devices and sensors are collecting data everywhere like in home, in car, at the office and in the manufacturing plant. Sending data and events through networks to some destination. Taking action based on the information and data communication with another machine $(\mathrm{m} 2 \mathrm{~m})$, Send a notification (sms, email, text), and talk to another system.

\section{HARDWARE OF IOT}

The hardware utilized in IoT systems includes devices for a remote dashboard, devices for control, servers, a routing or bridge device, and sensors. These devices manage key tasks and functions such as system activation, action specifications, security, communication, and detection to support-specific goals and actions. The core components of the IoT will be sensors and actuators, embedded processing, and connectivity and the cloud. Smart objects such as modern phones use sensors and actuators to interact with the real world. Embedded processing gives smart objects intelligence while connectivity and the cloud provide the means to communicate and store data. The IoT will ultimately evolve into a network of people, processes, data, and physical objects that intercommunicate using wireless protocols.

The most important hardware in IoT might be its sensors. These devices consist of energy modules, power management modules, RF modules, and sensing modules. The sensing module manages sensing through assorted active and passive measurement devices. RFID is a system in which there is a reader to read many tags as presented by Want et. al. [3] (2006). Machine-to-Machine (M2M) refers to the communications between computers, embedded processors, smart sensors, actuators and mobile devices (DYE, 2008). The use of M2M communication is increasing in the scenario at a fast pace. For instance, researchers predicted that, by 2014, there will be 1.5 billion wirelessly connected devices excluding mobile phones[4].

Arduino Uno is a microcontroller board based on 8-bit ATmega328P microcontroller. Along with ATmega328P, it consists of other components such as crystal oscillator, serial communication, voltage regulator, etc. to support the microcontroller. Arduino Uno has 14 digital input/output pins (out of which 6 can be used as PWM outputs), 6 analog input pins, a USB connection, A power barrel jack, an ICSP header and a reset button. Figure 3 shows hardware components of Arduino board.

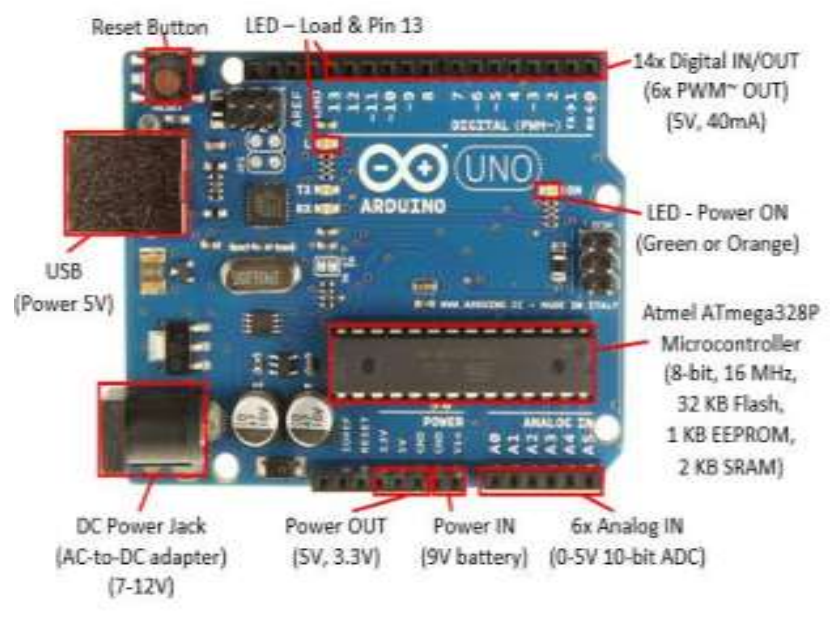

Fig. 3. Arduino Uno board hardware and its components

\section{APPLICATIONS OF IOT}

Today we can find many applications of IoT in almost all fields of society and technology. Wide variety applications are includes building and home automation, manufacturing, medical and healthcare systems, media environmental monitoring infrastructure management, energy management transportation, industry, and infrastructure, government and safety home and office health and medicine. Also IoT finds applications in marketing and content delivery improved advertising, air and water pollution extreme weather commercial farming intelligent product enhancements, dynamic response to market demands improved facility safety residential energy commercial energy, research, medical information distribution emergency care, environment and conditioning, health and safety. In industrial work, a machine can sense the work efficiency of the machine and work accordingly for maximum output. Smart homes where objects can communicate with each other like when there is no one in the home and unfortunately the owner forgot to lock the home then smart home will sense that there is no motion in the home and it will lock the home and send the unlock key to the owner 


\section{International Journal of Engineering Applied Sciences and Technology, 2019 Vol. 4, Issue 3, ISSN No. 2455-2143, Pages 153-156 \\ Published Online July 2019 in IJEAST (http://www.ijeast.com)}

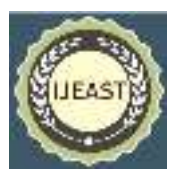

given by Moghaddam et. al. [5] (2015). The same application is smart water supply; if there is a leakage then the machine sensor will sense this and send the information to the server. It will help to stop the wastage of water [6] (2014). In this technology the objects are vehicles, which can communicate with another vehicle or the sensors around them. The main aspect of concern here is, there is no proper method to define the protocols because the object is moving and communicating with another moving object or with the sensors on the road side [7] (2016).

Wearables are experiencing highest demand in market. Wearable's which have embedded in sensors collect data from users and transmit the insight data to users, e.g. it covers health and fitness requirements. They are small in size, low power and highly energy efficient. Car connecting operation such as maintenance of the car and performing operation by its own are performed. Passenger's understanding the best comfort zone by accessing internet facility. Huge companies are working on this connected car solutions. Smart Cities, One of the interesting applications in IoT is smart city; it is creating curiosity in population. Surveillance, energy management, environment monitoring, water distribution, automated transportation and urban security are examples of smart cities. Agriculture: Supplying food to increasing population is one of the greatest demands. Government is helping farmers, by using research and advanced technology to increase their food production. IoT helps in sensing moisture in soil, controlling water usage for plant, and custom fertilizer. Day by day IoT is improving human life in a more convenient and simpler way, it is creating buzz, making people to experience new digital world never before. In future smart home will become as easier as smart phones. For example switching off air conditioner when no lives in home, unlock door for friends for temporary access when owner is not available, and opening door as owner enters etc are well known examples of IoT.

\section{CONCLUSION}

IoT is shows potential approaches to the world community and it works on various domains. Every object interacts digitally and prevents human monitoring the machineries and direct involvement. It is used in wide variety of applications such as smart cities, smart homes, agriculture, wearable's, and healthcare it also faces various challenges like security, privacy and compatibility.

This paper systematically focuses on new generation using IoT. Which gives idea on how today human beings are living based on technologies. IoT is developed based on internet this truth is objectively proved in this paper. Also this paper gives clarity to what is next after internet. Through a widely distributed, locally intelligent network of smart devices, the IoT has the potential to enable extensions and enhancements to fundamental services in transportation, logistics, security, utilities, education, healthcare and other areas, while providing a new ecosystem for application expansion. A concerted effort is required to move the industry beyond the early stages of market development towards maturity, driven by common understanding of the distinct nature of the prospectus. This market has diverse characteristics in the areas of service distribution, business and charging models, capabilities required to deliver IoT services, and the differing demands these services will place on mobile networks.

\section{REFERENCE}

[1] Memon, Azam Rafique, "An Electronic Information Desk System For Information Dissemination In Educational Institutions."

[2] I.F. Akyildiz, W. Su, Y. Sankarasubramaniam, E. Cayirci,(2002) Wireless sensor networks: a survey, Computer Networks 38, 9pp. 393-422).

[3] Want, R. (2006), An Introduction to RFID Technology. IEEE Pervasive Computing, 5, (pp.25-330.

[4] H. C. Chen, M. A. A. Faruque and P. H. Chou, (2016) "Security and privacy challenges in IoT-based machineto-machine collaborative scenarios," International Conference on Hardware/Software Codesign and System Synthesis (CODES+ISSS), Pittsburgh, PA, (pp.1-20).

[5] Anvari-Moghaddam, A., Monsef, H. and RahimiKian, A. (2015) Optima Smart Home Energy Management Considering Energy Saving and a Comfortable Lifestyle. IEEE Transactions on Smart Grid, 6, (pp324-3320).

[6] Prajakta Pande and Anand R. Padwalkar,(2014) "Internet of Things -A Future of Internet: A Survey", International Journal of Advance Research in Computer Science and Management Studies Research Article / Paper / Case Study Volume 2 , Issue 2 , (pg .354-3610).

[7] Y.Usha Devi, Dr. M.S.S.Rukmini, (2016) "IoT in Connected Vehicles: Challenges andIssues- A Review," International conference on Signal Processing, Communication, Power and Embedded System (SCOPES).

[8] Salonie Vyas, Umang Chaudhari, V. Chinmay Nandini, Bhushan Thakare, (2015) "State of the Art Literature Survey 2015 on RFID," International Journal of Computer Applications Volume 131 - No.8, (pp. 0975 8887)

[9] White Paper on "Machine-to-Machine Communication (M2M)"

[10] S. Riazul Islam, D. Kwak, M. Humaun Kabir, M. Hossain, and K.S. Kwak, (2015), "The internet of things for health care: a comprehensive survey," IEEE Access, vol. 3, (pp.678-708).

[11] Anusha Rahul, Gokul Krishnan G, Unni Krishnan H and Sethuraman Rao, (2015), "NEAR FIELD 
International Journal of Engineering Applied Sciences and Technology, 2019

Vol. 4, Issue 3, ISSN No. 2455-2143, Pages 153-156

Published Online July 2019 in IJEAST (http://www.ijeast.com)

COMMUNICATION (NFC) TECHNOLOGY: A SURVEY," International Journal on Cybernetics \& Informatics (IJCI) Vol. 4, No. 2.

[12] Handong Zhang and Lin Zhu, (2011), "Internet of Things: Key technology, architecture and challenging problems," IEEE International Conference on Computer Science and Automation Engineering, Shanghai, 2011, (pp. 507-512).

[13] Shashank Agrawal, Dario Vieira, (2013), "A survey on Internet of Things," Abakós, Belo Horizonte,v. 1, n. 2, , maio - ISSN:2316-9451 (pp. 78 - 95).

[14] Yinghui H., Guanyu L., (2010). Descriptive Models for Internet of Things. IEEE International Conference on Intelligent Control and Information Processing, Dalian, China, (pp. 483-486).

[15] Tongzhu Z., Xueping W., Jiangwei C., Xianghai L., Pengfei C., (2010) .Automotive recycling information management based on the internet of things and RFID technology. IEEE International Conference on Advanced Management Science (ICAMS), Changchun, China, page(s):620 - 622 . 